\title{
A Humanização nas Grades Curriculares de Cursos da Saúde de Universidades Públicas Paranaenses
}

\author{
The Humanization in the Undergraduate Curricular Grades of Health Courses in Paraná's \\ Public Universities
}

\section{La Humanización en las Grades Curriculares de Cursos de la Salud en las Universidades Públicas Paranaenses}

\author{
Debora Lydines Martins Corsino \\ Maíra Bonafé Sei ${ }^{1}$ \\ Universidade Estadual de Londrina
}

\begin{abstract}
Resumo
A humanização em saúde é uma política pública relativamente recente. Há ações que buscam fomentar a humanização tanto no cuidado quanto na formação em saúde, mas sem dados objetivos acerca da presença desse tema nas grades curriculares da graduação. Objetivou-se realizar um levantamento acerca da presença do tema "humanização" nas grades curriculares de cursos da área da saúde das universidades públicas do estado do Paraná, por meio de um estudo transversal e descritivo. Foram consultados documentos disponíveis on-line nos sites das instituições, verificando-se a presença do tema "humanização" nas ementas das disciplinas de cada curso. Percebeu-se que o tema estudado ainda é pouco abordado em disciplinas das graduações em saúde. Diante disso, apontou-se a predominância da perspectiva biológica, entre outros fatores, para debater a escassez de um conteúdo tão pertinente para o ensino do cuidado à saúde.

Palavras-chave: humanização em saúde, formação em saúde, pessoal de saúde, capacitação de recursos humanos em saúde
\end{abstract}

\begin{abstract}
Humanization in health is a relatively recent public policy. There are actions that seek to foster humanization in both health care and training, but with no objective data about the presence of this theme in the undergraduate curricular grids. This study aimed to present a cross-sectional and descriptive study about the presence of the theme "humanization" in undergraduate curricular grades of courses in the health area of public universities in the State of Paraná. We searched documents available on the institutions' websites, checking for the presence of the theme "humanization" in the menus of the disciplines of each course. We noticed that, the subject studied is still little approached in undergraduate courses disciplines in health. In view of this, we point out the predominance of the biological perspective, among other factors to discuss the scarcity of such relevant content in healthcare training.

Keywords: humanization in health, health education, health personnel, training of human resources in health
\end{abstract}

\section{Resumen}

La humanización en salud es una política pública relativamente reciente. Hay acciones que buscan fomentar la humanización tanto en el cuidado como en la formación en salud, pero sin datos objetivos acerca de la presencia de este tema en las grades curriculares de la graduación. Se objetivó realizar un relevamiento acerca de la presencia del tema "humanización" en las grades curriculares de cursos del área de salud en las universidades públicas del Estado de Paraná, a través de un estudio transversal y descriptivo. Se consultaron los documentos disponibles en línea en los sitios web de las instituciones, verificándose la presencia del tema "humanización" en los programas de las asignaturas de cada curso. Se percibió que el tema estudiado todavía es poco abordado en asignaturas de las graduaciones en salud. Ante ello, se subrayó la predominancia de la perspectiva biológica, entre otros factores para debatir la escasez de un contenido tan pertinente, para la enseñanza del cuidado a la salud.

Palabras-clave: humanización en salud, formación en salud, personal de salud, capacitación de recursos humanos en salud

\footnotetext{
${ }^{1}$ Endereço de contato: Departamento de Psicologia e Psicanálise da Universidade Estadual de Londrina, Rodovia Celso Garcia Cid- Pr 445- Km 380, Campus Universitário, Cx. Postal 10011, CEP 86057-970, Londrina, PR.
} 


\section{Introdução}

De acordo com Almeida e Chaves (2013), o termo humanização pode ser entendido como encontro de sujeitos e de suas subjetividades. Esse encontro é fundamento para o ato do cuidar, de dar assistência necessária ao indivíduo, em que uma relação saudável de aprendizagem recíproca é privilegiada. Além disso, humanizar o cuidado é valorizar todos os sujeitos envolvidos no processo de promoção de saúde, garantindo o protagonismo e a autonomia destes, com fortalecimento de vínculos, participação coletiva e cogestão (Brasil, 2004; 2010).

A prática de humanizar o cuidado à saúde emergiu dentro do Sistema Único de Saúde (SUS), haja vista que este carrega princípios e valores que privilegiam a dignidade humana, como: a universalidade, a integralidade, a equidade e a participação social (Brasil, 1988). O exercício da humanização surge em resposta à insatisfação de condições precárias de trabalho, que geravam pacientes mal recepcionados e profissionais desvalorizados (Pialarissi, 2017).

Desde a regulamentação da Constituição de 1988 e da fundação do SUS, a garantia de saúde passou a ser um direito de todos os cidadãos e um dever do Estado. Entretanto, em razão das falhas de investimento dos governantes e com a crise de contribuição e recolhimento da previdência social do Instituto Nacional de Seguro Social (INSS), a rede de saúde está em colapso (Santos, 2015).

A crise orçamentária gera um ambiente de trabalho com poucos recursos disponíveis para os profissionais exercerem sua prática com êxito. Além da escassez de recursos físicos, os profissionais lidam diariamente com a insuficiência de tempo para atender a todas as demandas dos pacientes usuários dos serviços. Essa "falta de tempo" desencadeia má qualidade dos atendimentos e o afastamento do profissional de seus pacientes. Assim, esse distanciamento e a coisificação das pessoas dentro dos hospitais removeram das relações interpessoais o respeito à autonomia e à solidariedade (Pialarissi, 2017; Neulls, 2016).

O ambiente hospitalar por si só é carregado de sofrimento, pois possui a doença como protagonista, e a hierarquia vertical, o autoritarismo, a soberania do saber médico e a ausência de direitos o tornam mais indigesto aos processos de trabalho. Os pacientes se converteram em objetos de estudo para a construção do saber científico e os profissionais passaram a ser vistos como engrenagens de uma máquina, na qual cada um exerce sua função. Esse processo de divisão de tarefas agiliza, sim, o atendimento, mas também concebe a alienação do trabalho, que afasta do profissional a responsabilidade na construção do todo (Rios, 2009; Neulls, 2016).

Esse processo de precarização do cuidado à saúde resultou em compromissos mínimos e ausência de cuidados aos usuários do sistema de saúde, o que desencadeou naturalização do sofrimento, diminuição da responsabilidade de cada sujeito pertencente à rede na produção de saúde, excesso de medicalização, entre outros pontos antagônicos ao tratamento humanístico. Em razão desse cenário, buscou-se resgatar valores como direitos, generosidade, respeito ao indivíduo e à sua subjetividade (Rios, 2009; Neulls, 2016). Casate e Corrêa (2012) retratam que a humanização emerge como tentativa de resolução de um estado de tensão e insatisfação, tanto dos pacientes como dos profissionais, a um montante de fatos que pode ser caracterizado como violência institucional na saúde, entendido como aplicação de abusos em instituições, possuindo a sociedade e o estado como cúmplices.

Com base nesse pressuposto, muitos hospitais, principalmente os públicos, iniciaram o desenvolvimento de práticas que receberam o nome de humanizadoras. Em seu início, es- 
sas ações se resumiam a atividades que tornavam o ambiente menos rude, com atividades lúdicas de lazer, arte e entretenimento e melhoramento dos aspectos físicos internos dos setores de atendimento. Tais atitudes não alteraram consideravelmente a disposição do trabaIho, mas serviram para amenizar o sofrimento que pacientes e profissionais padeciam com a estrutura falha do sistema de saúde. Com esse pontapé inicial, a prática foi ganhando corpo, desencadeando alterações substanciais na rotina, como partos humanizados com atenção à mulher, dieta personalizada, visitas livres, direito a acompanhante, entre outras (Rios, 2009).

Em 2000, surgiu o Programa Nacional da Assistência Hospitalar (PNHAH), que inseriu, nas discussões de âmbito hospitalar, princípios que deveriam nortear o cuidado pela perspectiva da humanização (Brasil, 2001). Em 2003, o programa passou por uma revisão e atualização e recebeu o nome de Política Nacional de Humanização (PNH) em saúde, embasando as práticas de todos os setores da saúde (Brasil, 2004). Discutir a prática humanística como política é relevante, pois garante-Ihe o destaque das diretrizes do sistema de saúde e, enquanto programa, apenas norteava tais diretrizes (Neulls, 2016).

Então, enquanto política, a PNH se apresenta como um conjunto de diretrizes transversais que norteia toda a atividade institucional que envolve usuários ou profissionais da saúde, em qualquer instância que será efetuada. Tais diretrizes apontam como caminhos: promoção da gestão participativa, mediante diálogo horizontal, entre profissionais, população e gestores; corresponsabilização destes no processo de promoção à saúde; consolidação das práticas do trabalho em equipe, estimulando práticas de comunicação que garantam acolhimento e escuta de acordo com a singularidade dos sujeitos; respeito à cultura e ao sistema organizacional da comunidade e do ambiente em que a unidade está inserida, entre outros (Brasil, 2004).

Pitombeira, Xavier, Barroso, e Oliveira (2016) descrevem as instituições formadoras como essenciais no processo de ensino-aprendizagem do modelo que privilegia a atenção integral aos usuários. Os autores afirmam que a metodologia de ensino deve perpassar o modelo hegemônico, buscando vincular o trabalho ao cotidiano da população atendida e remodelar as formações segundo essa perspectiva. Com esse viés atendido, os indivíduos envolvidos nesse processo - docentes, discentes, usuários, profissionais e gestores - ficam sujeitos à "produção de subjetividade, produção de habilidades técnicas e de pensamento" adequados à realidade e à prática do SUS (Pitombeira et al., 2016, p. 282).

Entretanto, para a inserção de um modelo de educação e saúde que privilegie o entendimento do ser humano enquanto biopsicossocial, é preciso romper com o modelo biomédico ainda vigente nas instituições, tanto nas de ensino superior que formam profissionais quanto nos próprios serviços de saúde (Silva, Muhl, \& Moliani, 2015; Ângulo-Tuesta et al., 2016). Além disso, a formação vinculada à realidade social dos sujeitos envolvidos gera estudantes e profissionais com autonomia e reflexão crítica, dispostos a problematizações e transformações constantes da realidade de ensino e trabalho (Pitombeira et al., 2016). Portanto, as instituições de ensino precisam pautar seus cursos de graduação em um entendimento interdisciplinar, visando, além dos conteúdos, a técnicas e teorias, perpassando também questões políticas, humanas, socioculturais, econômicas e tecnológicas da atualidade, para suprir as necessidades de formação para o SUS (Rosevics et al., 2014).

Em 2004, PNH apontou a necessidade e a urgência de inserir tais conteúdos nas formações dos profissionais da saúde. Entretanto, observa-se que a presença dessas discussões nos cursos superiores ainda é escassa e, quando ocorre, insuficiente (Silva et al., 2015; 
Almeida \& Chaves, 2013). Portanto, torna-se relevante investigar se as universidades estão abordando os pressupostos da humanização em meio aos graduandos e futuros profissionais da área da saúde e de que maneira estão realizando essa abordagem, no sentido de refletir sobre a formação ofertada. Assim, a presente pesquisa objetivou realizar um levantamento acerca da presença do tema "humanização" nas grades curriculares de cursos da área de saúde das universidades públicas paranaenses, para investigar o quanto as instituições têm se dedicado a incluir esse conteúdo nas formações.

Sabe-se que a formação superior é regulada por diversos instrumentos que compõem as leis de educação e ensino no Brasil, entre eles a Leis de Diretrizes e Bases (LDB) 9.394/96 (Brasil, 1996) e as Diretrizes Curriculares Nacionais (DCNs). Tais documentos assumem que, apesar de possuírem princípios que norteiam a construção dos conteúdos, as instituições de ensinos têm autonomia para adequar a proposta curricular de acordo com a realidade cultural e social da região na qual está inserida, a fim de possibilitar a formação de profissionais com diversas especialidades no país (Brasil, 1996).

Nesse sentido, as instituições de ensino superior (IES) produzem o projeto pedagógico do curso (PPC), para cada curso de graduação, evidenciando a relação com as DCNs, o histórico do curso e como este se estrutura, os princípios da formação, a grade curricular, entre outros elementos (Fernandes, 2016). Seixas (2014) aponta que, apesar de não haver normas para os PCCs, estes possuem uma regularidade. O autor apresenta os PCCs com um primeiro espaço dedicado aos princípios filosóficos e pedagógicos da instituição. Em um segundo espaço são delineadas as particularidades das ênfases oferecidas pela instituição e, por fim, há a formação profissional guiada pela matriz curricular, que descreve as atividades, os estágios e a grade curricular.

A grade curricular é composta das disciplinas do curso e das suas ementas, que, em forma de texto, expõem objetivamente os conteúdos centrais e norteadores que são ministrados e direcionam o docente na elaboração do programa da disciplina, que se modifica a cada ano. Seixas (2014) menciona que as ementas presentes no PPCs são "a orientação mais geral e permanente que uma disciplina pode ter" (p. 192), alterando-se apenas quando um novo projeto pedagógico é instalado. Portanto, a presente pesquisa se ateve a investigar as ementas, entendendo que estas fazem parte da base da construção do ensino da humanização nas instituições.

\section{Método}

Trata-se de uma pesquisa de estudo transversal de caráter descritivo. Com base nas grades curriculares, consultaram-se as ementas das disciplinas de cursos da saúde, das IES públicas do Paraná, para avaliar a presença do tema "humanização" na formação proposta por tais cursos.

\section{Procedimentos}

Inicialmente, foram consultados os sites das seguintes instituições públicas paranaenses: Universidade Estadual de Londrina (UEL), Universidade Estadual de Maringá (UEM), Universidade Estadual de Ponta Grossa (UEPG), Universidade Estadual do Oeste do Paraná (Unioeste), Universidade Estadual do Centro-Oeste (Unicentro), Universidade Estadual do 
Norte do Paraná (Uenp), Universidade Estadual do Paraná (Unespar) e Universidade Federal do Paraná (UFPR), a fim de verificar a disponibilidade on-line dos documentos (PPCs, ementários, matrizes curriculares, entre outros) que continham as grades curriculares vigentes dos cursos da saúde. Com os documentos obtidos, realizaram-se leituras das ementas de todas as disciplinas das séries ou períodos dos cursos da saúde, em busca do tema "humanização".

Entretanto, algumas universidades e cursos não disponibilizavam as ementas nos sites. Desta forma, em um segundo momento, realizou-se contato com os coordenadores de curso ou colegiado com uma carta de solicitação desses documentos. Com isso, obteve-se acesso às grades curriculares de mais alguns cursos e universidades e, por meio da leitura destas, procedeu-se à busca pelo tema "humanização".

Os documentos adquiridos e pesquisados foram armazenados com a ferramenta Google Drive, que possibilita manter os dados salvos on-line, e com o recurso de planilha também da ferramenta foi possível organizar os dados obtidos para melhor visualização dos resultados.

\section{Análise de Dados}

Para a análise de dados, buscou-se nas ementas de cada disciplina o termo "humanização", vinculado aos conteúdos que explicitamente se referiam à prática em saúde humanizada. Os cursos que tinham nas ementas a apresentação do termo atrelado a outras temáticas não foram incluídos nos resultados, visto que a abordagem do tema não se referia diretamente ao contexto da saúde. Com os resultados obtidos, realizaram-se exposição e comparação entre as propostas de grades das instituições, tecendo considerações sobre a frequência com que esse conteúdo é apresentado, discutindo a necessidade de pensar o processo de formação como primordial para a produção de profissionais de saúde humanizados.

Mesmo com uma busca on-line e o contato com as secretarias e colegiados de curso, vale ressaltar que não se obteve acesso às ementas de alguns cursos e universidades, portanto os dados destes não foram incluídos nos resultados. São eles: Educação Física e Enfermagem da Unespar, Enfermagem - Campus Foz do Iguaçu da Unioeste, Medicina - Campus Cascavel da Unioeste, Medicina e Nutrição - campus Francisco Beltrão da Unioeste, Fisioterapia e Saúde Coletiva - campus Matinhos da UFPR, Enfermagem, Farmácia, Fisioterapia, Medicina, Nutrição e Odontologia - campus Curitiba da UFPR.

\section{Resultados}

Com base nas consultas realizadas nos sites das universidades e no contato com algumas secretarias e coordenadorias de curso, totalizaram 37 cursos para análise das ementas, de sete IES públicas. Dos 37 cursos, sete são de Educação Física, seis de Enfermagem, cinco de Farmácia, cinco de Odontologia, quatro de Fisioterapia, quatro de Psicologia, três de Medicina, um de Nutrição, um de Fonoaudiologia e um de Terapia Ocupacional. Dos cursos incluídos na pesquisa, sete são da UEL, seis são da UEM, quatro são da Uenp, cinco são da UEPG, oito são da Unicentro, quatro são da Unioeste e três da UFPR.

Das 37 grades curriculares analisadas, dez abordam em algum momento o tema "humanização", atrelado aos conteúdos de saúde. Vale ressaltar que duas disciplinas encontradas são optativas, portanto nem todos os alunos formados nos cursos em questão têm acesso a elas. 
Dos cursos que abordam o tema "humanização" em algum momento nas ementas, cinco são do curso de Enfermagem, dois do curso de Farmácia, um do curso de Fisioterapia, um do curso de Psicologia e um de Terapia Ocupacional.

Em relação às instituições incluídas no estudo, dos dez currículos que abordam a temática estudada nesta pesquisa, UEL, UEPG, Unicentro e Unioeste possuem dois desses cursos, enquanto a UEPG e a UFPR, um cada uma.

Os cursos que apresentam disciplinas que abordam o tema na modalidade de disciplina obrigatória são Enfermagem - UEL, Enfermagem - Uenp, Enfermagem e Farmácia - UEPG, Enfermagem e Fisioterapia - Unicentro, Enfermagem e Farmácia - Unioeste. Os cursos que apresentam o tema na modalidade de disciplina optativa são Psicologia - UEL e Terapia Ocupacional - UFPR.

Dos cursos citados, as disciplinas que contemplam o tema humanização em saúde são Saúde da criança e do adolescente, Contribuições da Psicologia para o trabalho em saúde, Saúde da mulher, Práticas clínicas em alta complexidade, Assistência da enfermagem ao paciente crítico, Estágio de iniciação em ciências farmacêuticas, Farmácia hospitalar, Cuidados de enfermagem para pacientes em situações críticas, Fundamentos da Epidemiologia e Saúde Pública, Procedimentos farmacêuticos assistenciais, Sistematização da assistência de enfermagem, Saúde coletiva III e Cuidados paliativos.

É válido ressaltar que nos resultados expostos estão inseridos os cursos que abordam explicitamente o tema "humanização", atrelados ao contexto da saúde. Alguns cursos margeiam conceitos que condizem com a prática humanizada, mas não abordam o tema diretamente na ementa. Além disso, alguns cursos trabalham com a conceituação de humanização, mas em contextos não diretamente da saúde (exs.: Psicologia sócio-histórica e Psicologia do trabalho da UFPR), portanto esses dados também não foram incluídos nos resultados.

Além disso, é importante pontuar que nenhum curso utilizado neste estudo possui uma disciplina específica sobre humanização em saúde.

\section{Discussão}

De acordo com os dados coletados, notou-se que mesmo com uma Política Nacional de Humanização instaurada há mais de dez anos no campo da saúde, inserções do tema ainda são insuficientes nos currículos de formação. O modelo de saúde no Brasil ainda é pautado em um referencial voltado ao orgânico, vinculado às práticas que dividem biológico-social-psíquico, como instâncias que necessitam de cuidados individualizados (Rosevics et al., 2014; Silva et al., 2015; Fernandes, 2016).

Essa premissa apresenta-se como uma das dificuldades em incluir na formação de profissionais da saúde aspectos que perpassam o olhar biologizante do indivíduo. Entende-se que para romper com essa perspectiva de ensino, a instituição formadora precisa assumir a responsabilidade de oportunizar diversos espaços que fomentem o aprendizado prático do mundo real, de acordo com a demanda da população atendida pelos serviços da instituição (Casate \& Corrêa, 2012).

Além disso, é válido ressaltar que mesmo o tema constando na ementa e estar dentro do conteúdo programático, pode não ser suficiente, já que a metodologia e o contexto em que o conteúdo é abordado influem diretamente na apreensão do conhecimento do aluno (Casate \& Corrêa, 2012). Sobre isso, Campos (2013) afirma que "a formação deve estar as- 
sentada não no conhecimento de conteúdos estanques, abstratos e descontextualizados, mas no conhecimento operacionalizado, capaz de mediar a atuação em outras palavras" ( $p$. 109). Os métodos de ensino precisam ir além do tradicional, garantindo a maturação dos potenciais humanísticos de autonomia, participação e transformação. As discussões acerca da temática precisam ser direcionadas sob uma perspectiva humana, política e social, inserindo a realidade dos usuários e dos serviços de saúde no cotidiano dos estudantes (Biscarde, Pereira-Santos, \& Silva, 2014). A oferta dos conteúdos teóricos humanísticos com a vinculação prática aplicada não está presente nas práticas acadêmicas e, com isso, não se mostra eficaz. Assim, torna-se necessário investigar, com base nos resultados, quais os métodos utilizados nas instituições que possuem o tema nas ementas, para analisar sua efetividade (Almeida \& Chaves, 2013).

Ângulo-Tuesta et al. (2016) apontam que as formações em saúde precisam ofertar aos discentes o espaço de fala e construção do saber, criando um modelo de ensino-aprendizagem horizontal. Portanto, os docentes precisam romper com o modelo priorizado em suas próprias formações e nas instituições, que apresentam o saber como dicotômico, e oportunizar um espaço democrático do saber, onde estudantes e professores possuem potência para ensinar e aprender. Nesse sentido, as ciências médicas precisam fortalecer seu vínculo com as ciências humanas, para formar profissionais com senso crítico, ético e pautado em relações interpessoais de respeito, comunicação e responsabilidade social.

Além disso, observou-se que no curso de Enfermagem há maior frequência de disciplinas que abordam o tema. Essa questão é algo a ser pensado segundo a forma como o curso foi construído ao longo do tempo. A enfermagem é vista como uma profissão cuidadora e que estabelece maior contato e por mais tempo com o paciente. Não é raro perceber que se trata dos profissionais que ficam mais endurecidos com anos de profissão. Esses fatores levam a entender que a demanda do curso faz tais aspectos serem questionados e, assim, surgem alternativas para que os profissionais ocupem uma posição crítica e de constante reavaliação de sua prática (Almeida \& Chaves, 2013). Sobre isso, as autoras ainda elencam que o curso de enfermagem possui uma tradição técnica de formação assistencial, o que justifica os dados encontrados neste estudo.

Nesse sentido é interessante destacar em quais disciplinas o tema "humanização" se mostra presente. Três das disciplinas encontradas dizem respeito à assistência (Assistência da enfermagem ao paciente crítico, Procedimentos farmacêuticos assistenciais, Sistematização da assistência de enfermagem). Esse dado é relevante, pois PNH aponta a necessidade de propor modelos de atuação pautados na promoção de saúde e autonomia, e não apenas no assistencial. Além disso, quatro das disciplinas apontam a humanização apenas no contexto hospitalar e relacionado com o fim da vida (Farmácia hospitalar, Cuidados de enfermagem para pacientes em situações críticas, Práticas clínicas em alta complexidade, Cuidados paliativos). Entende-se que o cuidado nos hospitais necessita do olhar humanizado, entretanto é perigoso restringir a política apenas nesse viés, visto que humanizar também inclui intervenções dos setores de atenção básica. Duas outras disciplinas apresentam o tema atrelado a intervenções para populações específicas, como crianças, adolescentes e mulheres, mostrando, sim, uma discussão pertinente, porém esta necessita da mesma atenção que o item anterior para não restringir a prática apenas a esses contextos. Por fim, quatro disciplinas aparentam fornecer discussões sobre humanização mais amplas e direcionadas ao trabalho 
pautado em autonomia do usuário, mediado pela comunicação e relação horizontal, como: Estágio de iniciação em ciências farmacêuticas, Contribuições da psicologia para o trabalho em saúde, Fundamentos da epidemiologia e saúde pública, Saúde coletiva III.

Outro aspecto a ser discutido se refere às dificuldades institucionais e políticas das universidades públicas em propor, aprovar e colocar em vigor um novo currículo. Em geral, o corpo docente de um curso é constituído de profissionais que possuem linhas de formação e pesquisas específicas, logo o currículo da graduação se embasa nesses aspectos. Além disso, os trâmites institucionais e todas as instâncias nas quais a proposta de um novo currículo deve ser apreciada tornam o processo lento, demorado e desgastante. Ambos os fatores podem ter influência direta na não inclusão de práticas humanísticas nos conteúdos ministrados aos discentes, lembrando que apenas IES públicas foram investigadas.

Entretanto, é importante considerar que os graduandos podem ter acesso ao tema por outros meios. Os docentes possuem liberdade para reformular o programa e o cronograma, inserindo o tema nos conteúdos ministrados para além das informações disponíveis da ementa que possui caráter mais sintético e perene. Todavia, quando esse docente é remanejado, a prática do ensino do tema pode se perder, pois como a abordagem do conteúdo não é prerrogativa institucional, outros docentes não possuem obrigatoriedade em abordar o tema.

Outra alternativa se apresenta por meio dos projetos de ensino, pesquisa e extensão que se propõem a estudar a humanização em saúde. Biscarde et al. (2014, p. 177) afirmam que, para as formações acadêmicas causarem reais impactos e modificações na visão do estudante acerca de sua atuação profissional, é fundamental reformular a concepção de "aquisição, transmissão e difusão de conhecimentos" como metodologia de aprendizagem. Nessa perspectiva, é a instituição de ensino a responsável por propor modelos mais abertos e alinhados aos processos culturais, científicos e educativos. Tais autores propõem a extensão universitária como uma alternativa para promover a comunicação entre a universidade e o meio em que está inserida, além de possibilitar o desenvolvimento de um trabalho pautado em um modelo crítico-reflexivo, com destaque nas relações sociais. Ainda sugerem que iniciativas extensionistas devem ser intrínsecas às atividades pedagógicas e de trabalho universitárias e, porventura, se tornar requisito obrigatório nas formações, já que preenchem muitas lacunas que as disciplinas obrigatórias deixam abertas.

É notório que as perspectivas pedagógicas e de trabalho nas formações acadêmicas têm sofrido modificações. Sabe-se que novos espaços de formação e diferentes entendimentos sobre homem e suas condições de saúde têm emergido na sociedade. Mas ainda há um longo caminho a ser percorrido para que as formações e os atendimentos nas esferas da saúde alcancem o ideal de integração e comunicação efetivas entre docentes, discentes, usuários, trabalhadores e gestores.

Entende-se que o presente estudo possui algumas limitações, pois os conteúdos das ementas não foram analisados de forma interpretativa, buscando apenas o termo "humanização" explícito. Contudo, há um questionamento importante: já que a prática da humanização é fundamentada em uma política, por que a grade apresenta os conteúdos da política, mas não é abordada diretamente? No que diz respeito à coleta sobre a temática, esta foi realizada apenas nas grades curriculares dos cursos, portanto sugere-se para pesquisas futuras a análise das DCNs, dos PCCs, dos programas das disciplinas, dos cursos e instituições citadas, para avaliar se estes apontam alguma menção ao tema humanização. Além disso, também seria pertinen- 
te pesquisar com os docentes e discentes se a humanização é abordada e de que forma, para investigar se a produção de saber na prática tem ultrapassado as limitações dos documentos.

\section{Conclusões}

Tendo em vista os dados coletados e analisados, concluiu-se que a presença do tema humanização nos conteúdos obrigatórios de graduações em Saúde das IES públicas do Paraná é baixa. Apesar de a PNH ser considerada uma política do SUS e regulamentar o ensino da humanização em saúde há mais de dez anos, os cursos públicos da área da saúde no Paraná ainda não se adequaram à proposta dessa política. Portanto, este estudo aponta a necessidade de desenvolvimento de novas pesquisas que verifiquem se as hipóteses levantadas para os resultados obtidos são válidas. Além disso, são pertinentes estudos que investiguem quais as causas de um conteúdo necessário na prática profissional em saúde ainda não ser ministrado de forma eficaz nas graduações em saúde do estado do Paraná.

\section{Referências}

Almeida, D. V., \& Chaves, E. C. (2013). O ensino da humanização nas disciplinas dos cursos de graduação em enfermagem. Investigación y Educación en Enfermaría, 31(1), 44-53. Disponível em http://www.scielo.org.co/scielo. php?pid=S0120-53072013000100006\&script=sci_arttext\&tlng=pt

Ângulo-Tuesta, A. J.; Parreira, C. M. S. F.; Castro, C.; Queiroz, E.; Jesus, K.; Kadri, M. R., \& Pinheiro, R. M. (2016). Formação em saúde: Nova política. Novas modelagens? In R. B. Ceccim, J. A. Kreut, J. D. P. Campos, F. S. Culau, L. A. F. Wottrich, \& L. L. Kessler (Orgs.), Informes da Atenção Básica (pp. 221-248). Porto Alegre: Rede Unida.

Biscarde, D. G. S., Pereira-Santos, M., \& Silva, L. B. (2014). Formação em saúde, extensão universitária e Sistema Único de Saúde (SUS): Conexões necessárias entre conhecimento e intervenção centradas na realidade e repercussões no processo formativo. Interface: Comunicação, Saúde e Educação, 18(48), 177-186. doi: http://dx.doi. org/10.1590/1807-57622013.0586

Brasil. (1996). Lei 9.394/96, de 20 de dezembro de 1996. LDB- Leis de Diretrizes e Bases da Educação Nacional. Estabelece as diretrizes e bases da educação nacional (8a ed. atualizada em 08/05/2013). Disponível em http://www.planalto.gov.br/ccivil_03/LEIS/L9394.htm

Brasil. (1988). Constituição da República Federativa do Brasil. Disponível em https://www2. senado.leg.br/bdsf/bitstream/handle/id/518231/CF88_Livro_EC91_2016.pdf

Brasil. (2001). Ministério da Saúde. PNHAH-Programa Nacional de Humanização da Assistência Hospitalar. Disponível em http://bvsms.saude.gov.br/bvs/publicacoes/pnhah01.pdf

Brasil. (2004). Ministério da Saúde. HumanizasuS. Política Nacional de Humanização. Disponível em http://bvsms.saude.gov.br/bvs/publicacoes/humanizasus_2004.pdf

Brasil. (2010). Ministério da Saúde. HumanizaSUS: Documento base para gestores e trabalhadores do SUS. Disponível em http://bvsms.saude.gov.br/bvs/publicacoes/ humanizasus_documento_gestores_trabalhadores_sus.pdf

Campos, E. B. V. (2013). Considerações sobre as implicações das Diretrizes Curriculares na formação do estagiário em Psicologia. Psicologia: Ensino \& Formação, 4(2), 100-118. Disponível em http://pepsic.bvsalud.org/scielo. php? script=sci_arttext\&pid=S2177-20612013000200007\&lng=pt\&nrm=iso\&tlng=pt 
Casate, J. C., \& Corrêa, A. K. (2012) A humanização do cuidado na formação dos profissionais de saúde nos cursos de graduação. Revista da Escola de Enfermagem da USP, 46(1), 219226. doi: http://dx.doi.org/10.1590/S0080-62342012000100029

Fernandes, S. R. F. (2016) Psicologia e formação generalista: Do currículo mínimo às diretrizes curriculares (Dissertação de Mestrado, Universidade Federal do Rio Grande do Norte, Natal, RN, Brasil). Disponível em https://repositorio.ufrn.br/jspui/handle/123456789/22362

Neulls, T. F. (2016) Humanização em saúde frente ao processo de precarização do trabalho: Análise acerca da Política Nacional de Humanização da Saúde no âmbito do SUS (Dissertação de Mestrado, Universidade Federal do Maranhão, São Luís, MA, Brasil). Disponível em https://tedebc.ufma.br/jspui/handle/tede/813

Pialarissi, R. (2017) Precarização do trabalho. Revista de Administração em Saúde, 17(66). Disponível em http://cqh.org.br/ojs-2.4.8/index.php/ras/article/view/11/21

Pitombeira, D. F., Xavier, A. S., Barroso, R. E. C., \& Oliveira, P, R. S. (2016). Psicologia e a formação para a saúde: Experiências formativas e transformações curriculares em debate. Psicologia: Ciência e Profissão, 36(2), 280-291. doi: http://dx.doi.org/10.1590/1982-3703001722014 Rios, I. C. (2009). Humanização: A essência da ação técnica e ética nas práticas de saúde. Revista Brasileira de Educação Médica, 33(2), 253-261. doi: http://dx.doi.org/10.1590/ S0100-55022009000200013

Rosevics, L., Aguiar, D. A., Borges, C. R., Hasegawa Filho, R., Yamashita, T. S., Manchack, A. C., \& Azevedo, V. F. (2014). ProCura - a arte da vida: Um projeto pela humanização na saúde. Revista Brasileira de Educação Médica, 38(4), 486-492. doi: http://dx.doi.org/10.1590/ S0100-55022014000400010

Santos, M. A. R. (2015). Direito a saúde e o financiamento do SUS (Trabalho de Conclusão de Curso, Universidade Tiradentes [UNIT], Estância, SE, Brasil). Disponível em http://openrit. grupotiradentes.com/xmlui/handle/set/1706

Seixas, P. S. (2014). A formação graduada em psicologia no Brasil: Reflexão sobre os principais dilemas em um contexto pós-DCN (Tese de Doutorado, Universidade Federal do Rio Grande do Norte, Natal, RN, Brasil). Disponível em http://repositorio.ufrn.br:8080/jspui/ bitstream/123456789/17401/1/PabloSS_TESE.pdf

Silva, L. A., Muhl, C., \& Moliani, M. M. (2015). Ensino médico e humanização: Análise a partir dos currículos de cursos de medicina. Psicologia Argumento 33(8), 298-309. doi: https:// dx.doi.org/10.7213/psicol.argum.33.080.A006

Recebido em: 11/05/2017 Última revisão: 09/10/2017

Aceite final: 09/10/2017

\section{Sobre os autores:}

Debora Lydines Martins Corsino - Psicóloga e residente em Saúde da Família, pela Universidade Estadual de Londrina.E-mail: debora_lydines@hotmail.com

Maíra Bonafé Sei - Psicóloga, Mestre, Doutora e Pós-Doutora em Psicologia Clínica-IP-USP. Professora Adjunta do Departamento de Psicologia e Psicanálise da Universidade Estadual de Londrina. E-mail: mairabonafe@hotmail.com 